\title{
Characterization of the novel PDE9 inhibitor BAY 73-669 I using a cGMP reporter cell line
}

\author{
Frank Wunder*1, Adrian Tersteegen ${ }^{2}$ and Martin Hendrix ${ }^{3}$
}

\author{
Address: ${ }^{1}$ Institute of Cardiovascular Research, Bayer HealthCare, 42096 Wuppertal, Germany, ${ }^{2}$ Molecular Screening Technology, Bayer \\ HealthCare, 42096 Wuppertal, Germany and '3nstitute of Medicinal Chemistry, Bayer HealthCare, 42096 Wuppertal, Germany \\ Email: Frank Wunder* - frank.wunder@bayerhealthcare.com \\ * Corresponding author
}

from 2nd International Conference of cGMP Generators, Effectors and Therapeutic Implications

Potsdam, Germany, 10-12 June, 2005

Published: 16 June 2005

BMC Pharmacology 2005, 5(Suppl I):P64 doi:I0.I I86/I47|-22 I0-5-SI-P64

Cyclic nucleotide-specific phosphodiesterases (PDEs) play an essential role in signal transduction by regulating the intracellular levels of cAMP and cGMP and, therefore, are important pharmacological targets. They have been classified into eleven families based on their substrate specificity, regulation and pharmacology.

Phosphodiesterase 9 (PDE9) is a novel PDE family which has been identified and characterized very recently. PDE9 is highly specific for cGMP and not inhibited by the nonselective phosphodiesterase inhibitor 3-isobutyl-1methyl-xanthine (IBMX).

We report here the generation and characterization of a stably transfected PDE9 CHO cell line, additionally expressing soluble guanylate cyclase (sGC), the olfactory cyclic nucleotide-gated cation channel CNGA2 and the photoprotein aequorin. In this cell line, intracellular cGMP levels can be monitored in real-time via aequorin luminescence induced by $\mathrm{Ca}^{2+}$ influx through CNGA2, acting as the intracellular cGMP sensor. This simple and sensitive assay system can be used for the characterization of the cellular activity of PDE9 inhibitors and for the search of new PDE9 inhibitors by ultra-high-throughput screening.

We used the recombinant PDE9 cell line to identify and characterize the cellular effects of the selective PDE9 inhibitor BAY 73-6691 $\left(\mathrm{IC}_{50}=55 \mathrm{nM}\right)$. This compound is in preclinical development for both as cognition enhancer and for the treatment of Alzheimer's disease.

BAY 73-6691 alone did not significantly increase basal cGMP levels in this experimental setting. However, in combination with submaximal stimulating concentrations of the sGC activator BAY 58-2667 or the sGC stimulator BAY 41-2272, BAY 73-6691 induced concentrationdependent luminescence signals and intracellular cGMP increases. BAY 73-6691 potentiated the cGMP signals generated by these sGC activating compounds and induced leftward shifts of the corresponding dose-response curves.

In summary, this assay system allows the real-time cGMP detection within living cells and makes it possible to screen for compound interaction with the NO/cGMP system, as exemplified here with the PDE9 inhibitor BAY 736691 . 